\title{
2-D Analysis of Leakage in Printed-Circuit Lines Using Discrete Complex-Images Technique
}

\author{
Joaquín Bernal, Francisco Mesa, Member, IEEE, and Francisco Medina, Senior Member, IEEE
}

\begin{abstract}
The mixed-potential integral equation is combined with the discrete complex-images technique to analyze the complete spectrum of multilayered printed transmission lines. A relevant contribution of the present two-dimensional approach is its ability to study both the bound and leaky regimes in a very simple, systematic, and efficient way. Since the analysis is carried out in the spatial domain, this method makes it possible to analyze the leakage phenomenon for structures with nonzero-thickness conductors. Efficient quasi-analytical techniques are employed to solve the integral equation.
\end{abstract}

Index Terms-Complex images, leaky modes, mixed-potential integral equation, printed-circuit lines.

\section{INTRODUCTION}

$\mathbf{I}$ NTEGRAL-EQUATION methods are widely recognized as very efficient tools for studying the propagation characteristics of printed-circuit lines. Often, these integral equations are posed in the spectral domain since the Green's functions of the layered medium are only known in closed-form in this domain [1]. Thus, many works have shown the efficiency of the spectral-domain approach (SDA) to compute the propagation parameters of both bound and/or leaky modes [2]-[5]. Nevertheless, a well-known limitation of the SDA is its inadequacy to deal properly with general nonzero thickness conductors. A possible alternative to the SDA, able to treat with nonzero-thickness conductors, is found when the corresponding integral equation is directly solved in the spatial domain [6]. This technique requires to compute the spatial-domain Green's functions from their spectral counterparts through Fourier-transform inversion. Numerically efficient integration schemes have been used in the past to perform that task [7]. However, a powerful technique proposed at the end of the 1980s, i.e., the discrete complex-images technique (DCIT) [8], has become, in a variety of versions [9]-[11], the standard technique to obtain spatial-domain Green's functions for layered structures. That technique, originally intended for the analysis of three-dimensional (3-D) planar circuits and antennas, has been recently adapted to deal with two-dimensional (2-D) guiding structures, including strip-like [12], coplanar waveguide [13], and arbitrary cross-sectional conductors [14]. The relevance of a fast generation of the Green's function in

Manuscript received April 4, 2001. This work was supported in part by the Comisión Interministerial de Ciencia y Tecnología, Spain, under Project TIC980630, and by Junta de Andalucía.

J. Bernal is with the Department of Applied Physics III, University of Seville, 41092 Seville, Spain.

F. Mesa is with the Department of Applied Physics I, University of Seville, 41012 Seville, Spain (e-mail: mesa@us.es).

F. Medina is with the Department of Electronics and Electromagnetism, University of Seville, 41012 Seville, Spain (e-mail: medina@us.es).

Publisher Item Identifier 10.1109/TMTT.2002.801320.
2-D problems is relatively higher than in 3-D cases because the CPU time devoted to solve the final system of linear equations is usually negligible in the 2-D situation and the Green's functions must be generated many times for different values of the unknown propagation constant.

Although [12]-[14] could deal with a great variety of multiconductor and multilayer structures, they were purposely restricted to study only the bound regime. The important question posed by the existence of both surface and space leaky-wave solutions has been often treated in the frame of the SDA [3], [5] in such a way that only zero-thickness strip-like or slot-like structures have been considered in depth. Moreover, accounting for leakage in the frame of SDA requires rather sophisticated phys$\mathrm{ical} /$ mathematical reasoning to properly choose the integration paths that moment-method spectral integrals have to run along. The same reasoning has to be used if the spatial-domain Green's function is obtained by means of a direct integration in the spectral domain [7]. Thus, the aim of this paper is to show how to extend the method proposed in [12] and [14] to also deal with the leaky regime. This new approach turns out to be very simple and numerically very efficient for strictly planar structures and capable of dealing with nonplanar conducting structures. These valuable features can be also advantageously used for studying the practical excitation of leaky modes [15], [16] since this 3-D problem involves as one of its crucial steps the computation of the reaction integrals appearing in the 2-D case.

\section{Formulation OF THE PROBlem}

The spatial-domain mixed-potential integral equation (MPIE) will be applied to study the wave propagation in printed-circuit lines such as that shown in Fig. 1. The required kernel of the MPIE, namely, the spatial-domain Green's functions associated with the scalar and vector potentials [6], have to be obtained from their corresponding spectral versions. This calculation implies to perform Fourier transform inversions of the following general type:

$$
G\left(\left|x-x^{\prime}\right|, z, z^{\prime}\right)=\frac{1}{2 \pi} \int_{-\infty}^{\infty} \tilde{G}\left(k_{z 0} ; z, z^{\prime}\right) e^{-j k_{x}\left|x-x^{\prime}\right|} d k_{x}
$$

where

$$
k_{z 0}=\sqrt{k_{0}^{2}-k_{x}^{2}-k_{y}^{2}}
$$

with $k_{x}$ being the transverse wavenumber, $k_{0}$ being the freespace wavenumber, and $k_{y}=\beta-j \alpha$ being the assumed propagation constant. (Note that $k_{z 0}$ has been used instead of $u_{0}$ with the purpose of setting all these variables as wavenumbers; 


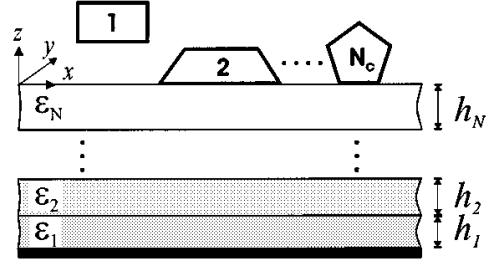

Fig. 1. Cross section of the structure under study.

$k_{z 0}=-j u_{0}$, where $u_{0}$ is the variable used in [14] and other papers.) It can be checked that the spectral-domain Green's functions of the layered structure are actually functions of $k_{z 0}$ provided that the media are isotropic and/or uniaxial anisotropic dielectric. Writing these functions in this convenient way (instead of as functions of $k_{x}$ ) is very relevant as it will become apparent later.

The DCIT can now be advantageously used to avoid the arduous computational effort involved in a direct numerical integration of (1). The efficiency of this approach basically lies in the fact that the spatial-domain counterpart of an spectral exponential term is known in closed form thanks to a 2-D version of the Sommerfeld identity. This idea has been successfully applied in [12]-[14] to characterize bound modes. Our purpose here is to generalize that formulation to also account for surface- and space-wave leaky modes. This generalization requires a previous treatment of the singularities (branch points and poles) of the spectral Green's functions since these singularities are directly related to radiation in the form of space and surface waves [17]. Considering that the underlying idea of the DCIT is to find a sum of complex exponential functions that fits properly $\tilde{G}$ and each exponential term is a complex analytical function, it is then crucial in this method to find that part of the Green's function that is actually analytic (i.e., free of singularities) within the range of interest. In this sense, it is important to note that, as a function of $k_{z 0}, \tilde{G}\left(k_{z 0}\right)$ is not a multivalued function because it does not show branch points in the complex $k_{z 0}$ plane. Nevertheless, $\tilde{G}\left(k_{z 0}\right)$ is still meromorphic since it does have poles, which should be extracted out in order to isolate the analytic part of $\tilde{G}\left(k_{z 0}\right)$. In fact, $\tilde{G}\left(k_{z 0}\right)$ has an infinite number of poles at $k_{z 0}= \pm k_{z p}$, where

$$
k_{z p}^{2}=k_{0}^{2}-\kappa_{p}^{2}
$$

with $\kappa_{p}$ being the wavenumber of the $p$ th mode of the grounded layered substrate. Despite the existence of infinite poles, in most practical cases, it is only necessary to extract out those poles appearing on the real axis, i.e., the poles associated with the above-cutoff surface waves of the grounded layered substrate [14]. For convenience, the quasi-static contribution should be also extracted out explicitly, as originally reported in [9] and later, in a 2-D context, in [12].

The combined MPIE-DCIT approach was used in [14] to study the bound regime in structures with nonplanar conductors. This approach will be briefly outlined here in order to introduce the nontrivial changes required to extend the method for dealing with the leaky regime. When nonplanar conductors are considered, the kernel of the integral equation contains several terms of the dyadic magnetic vector-potential Green's function and some derivatives plus the term associated to the scalar-poten- tial Green's function [14]. Thus, the Green's function problem can be reduced to obtaining the space-domain version of the following generic Green's function:

$$
\tilde{G}\left(k_{z 0}\right)=K\left[\frac{e^{-j k_{z 0}\left(z-z^{\prime}\right)}}{j k_{z 0}}+\tilde{S}\left(k_{z 0}\right)\right]
$$

where $K$ is a frequency-dependent constant and

$$
\widetilde{S}\left(k_{z 0}\right)=\tilde{G}^{\prime}\left(k_{z 0}\right) e^{-j k_{z 0}\left(z+z^{\prime}\right)}
$$

with $\tilde{G}^{\prime}\left(k_{z 0}\right)$ being the spectral Green's function for $z \equiv z^{\prime}=0$ (apart from $K$ ).

The spectral function $\widetilde{S}\left(k_{z 0}\right)$ can be split into the following three different contributions:

$$
\tilde{S}\left(k_{z 0}\right)=\tilde{S}_{0}\left(k_{z 0}\right)+\tilde{S}_{P}\left(k_{z 0}\right)+\frac{1}{k_{z 0}} \widetilde{S}_{I}\left(k_{z 0}\right)
$$

where $\widetilde{S}_{0}$ accounts for the quasi-static part

$$
\tilde{S}_{0}\left(k_{z 0}\right)=\lim _{k_{z 0} \rightarrow \infty} \tilde{S}\left(k_{z 0}\right)=C \frac{e^{-j k_{z 0}\left(z+z^{\prime}\right)}}{j k_{z 0}}
$$

(with $C$ being a known constant); $\widetilde{S}_{P}$ represents the contribution of the $N_{0}$ significant surface-wave terms

$$
\widetilde{S}_{P}\left(k_{z 0}\right)=e^{-j k_{z 0}\left(z+z^{\prime}\right)} \sum_{p=1}^{N_{0}} \frac{2 R_{p} k_{z p}}{k_{z 0}^{2}-k_{z p}^{2}}
$$

where $k_{z p}$ is the pole associated with the $p$ th surface wave and $R_{p}$ is its residue. $\tilde{S}_{I}$ is the remaining quasi-analytical part that can be accurately expanded as the following sum of complex exponential functions:

$$
\widetilde{S}_{I}\left(k_{z 0}\right)=k_{z 0}\left(\tilde{S}-\tilde{S}_{0}-\tilde{S}_{P}\right) \approx \sum_{i=1}^{N_{m}} a_{i} e^{-j k_{z 0}\left(\gamma_{i}+z+z^{\prime}\right)}
$$

where $a_{i}$ and $\gamma_{i}$ are, respectively, the amplitude and argument of each exponential term that expands the part of $\widetilde{S}_{I}$ not depending of $z$ and $z^{\prime}$.

\section{A. Treatment of $\tilde{S}_{P}\left(k_{z 0}\right)$}

The spatial-domain version of $\tilde{S}_{P}$ can be written as $S_{P}\left(\left|x-x^{\prime}\right|, z, z^{\prime}\right)=-\frac{1}{\pi} \sum_{p=1}^{N_{0}} R_{p} k_{z p} I_{p}\left(\left|x-x^{\prime}\right|, z, z^{\prime} ; k_{y}\right)$ where $I_{p}$ is given by

$$
I_{p}=\int_{-\infty}^{\infty} \frac{e^{-j k_{z 0}\left(z+z^{\prime}\right)}}{k_{x}^{2}-k_{x p}^{2}} e^{-j k_{x}\left|x-x^{\prime}\right|} d k_{x}
$$

with $k_{x p}^{2}=\kappa_{p}^{2}-k_{y}^{2}$. For the nonplanar case, the above integral and its derivative with respect to $\left|x-x^{\prime}\right|$ can be efficiently computed using integration contour techniques, as explained in the Appendix. However, for the planar case $\left(z \equiv z^{\prime}=0\right)$, (11), which will be denoted as $I_{p}^{0}\left(k_{y}\right)$, can be obtained in closed form. If relationship [18, (4.91)] is now adapted and analytically extended to make $I_{p}^{0}\left(k_{y}\right)$ continuous in the entire complex $k_{y}$ plane, $I_{p}^{0}\left(k_{y}\right)$ can be conveniently expressed as

$$
I_{p}^{0}\left(k_{y}\right)= \begin{cases}\frac{e^{-\sqrt{k_{y}^{2}-\kappa_{p}^{2}}\left|x-x^{\prime}\right|}}{\sqrt{k_{y}^{2}-\kappa_{p}^{2}}}, & \operatorname{Im}\left(k_{x p}\right)<0 \\ \frac{e^{-j \sqrt{\kappa_{p}^{2}-k_{y}^{2}}\left|x-x^{\prime}\right|}}{j \sqrt{\kappa_{p}^{2}-k_{y}^{2}}}, & \operatorname{Im}\left(k_{x p}\right)>0\end{cases}
$$




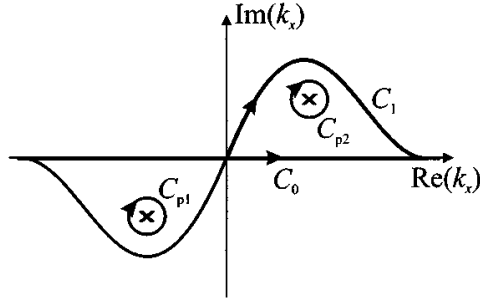

Fig. 2. Paths of integration in the complex $k_{x}$-plane. For simplicity, only a pair of surface-wave poles are shown $(\mathbf{x})$.

in order to emphasize the nature of the associated field. From a physical point-of-view, the $\operatorname{Im}\left(k_{x p}\right)<0$ case implies that the fields associated with the above-cutoff $p$ th surface wave decay exponentially in the transverse direction. Taking all the surface-wave terms in this way would be consistent with the bound regime. The surface-wave leaky regime is accounted for by the $\operatorname{Im}\left(k_{x p}\right)>0$ case, which gives the characteristic exponentially growing-field behavior in the transverse direction [17]. Taking the second choice for some of the above-cutoff surface waves, the resulting leaky regime would account for a mode radiating in the form of only those surface waves thus considered.

The above results can be related to the SDA by means of contour integration techniques. If, for simplicity, only one surface wave is assumed to be above cutoff, the bound-regime result in (12) is equivalent to having used the conventional real-axis integration path when performing the Fourier-transform inversion (path $C_{0}$ in Fig. 2). The surface-wave leaky-regime result can be viewed as a consequence of employing an integration contour detouring around the poles as path $C_{1}$ in Fig. 2. Taking into account that the integrand in (11) has no branch points, integration along path $C_{0}$ is equivalent to integration along path $C_{p 1}$ in Fig. 2, whereas the integration path $C_{1}$ is equivalent to the integration path $C_{p 2}$. Therefore, switching between $C_{0}$ and $C_{1}$ paths simply implies changing the pole that is captured by the integration path. This switch is equivalent to choose the pole according to the sign of its imaginary part.

The above discussion has shown that the selection of the regime to be deal with (bound or surface-wave leakage) is simply imposed by the proper choice in (12). Following the theory given in [15], the moment-method determinant function whose zeros are the propagation constants of the line defines a Riemann surface in the longitudinal wavenumber complex $k_{y}$-plane with multiple branch points. Thus, the choice made in $I_{p}^{\mathrm{O}}\left(k_{y}\right)$ can be related to the proper/improper sheet that the propagation constant $k_{y}$ will be located on when searching for the roots of the dispersion equation of the line.

\section{B. Treatment of $\tilde{S}_{I}\left(k_{z 0}\right)$}

Taking into account the complex exponential expansion (9), the space-domain contribution associated with $\tilde{S}_{I}^{\prime}=\tilde{S}_{I} / k_{z 0}$ can be expressed as

$$
S_{I}^{\prime}\left(\left|x-x^{\prime}\right|, z, z^{\prime}\right)=\sum_{i=1}^{N_{m}} a_{i} \Gamma_{i}\left(\left|x-x^{\prime}\right|, z, z^{\prime}\right)
$$

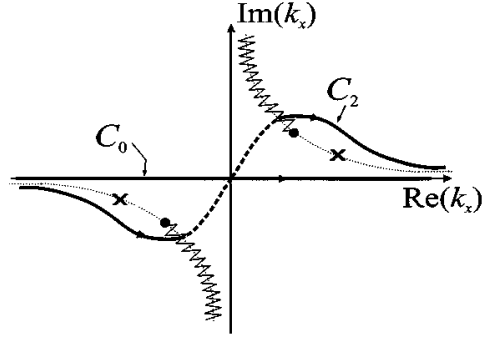

Fig. 3. Integration paths $C_{0}$ and $C_{2}$ in the complex $k_{x}$-plane. Integration paths on the proper/improper sheet with respect to the $\pm k_{x 0}$ branch points are display in solid/dashed lines. For simplicity, only a pair of surface-wave poles are shown $(x)$

where

$\Gamma_{i}\left(\left|x-x^{\prime}\right|, z, z^{\prime}\right)=\int_{-\infty}^{\infty} \frac{e^{-j k_{z 0}\left(\gamma_{i}+z+z^{\prime}\right)}}{4 \pi j k_{z 0}} e^{-j k_{x}\left|x-x^{\prime}\right|} d k_{x}$.

Following a similar rationale as that previously used for $I_{p}^{0}$, each of the integral representations given by the inverse Fourier transform $\Gamma_{i}$ will be expressed in an appropriate closed form. Thus, relationship [18, (4.156)] is now adapted and analytically continued in the entire $k_{y}$ plane to write

$$
\Gamma_{i}= \begin{cases}\frac{1}{2 \pi} K_{0}\left(\sqrt{k_{y}^{2}-k_{0}^{2}} \xi_{i}\right), & \operatorname{Im}\left(k_{x 0}\right)<0 \\ \frac{1}{4 j} H_{0}^{(2)}\left(\sqrt{k_{0}^{2}-k_{y}^{2}} \xi_{i}\right), & \operatorname{Im}\left(k_{x 0}\right)>0\end{cases}
$$

where $\pm k_{x 0}= \pm \sqrt{k_{0}^{2}-k_{y}^{2}}$ are the branch points of the Green's function in the complex $k_{x}$-plane, $\xi_{i}=\sqrt{\left(x-x^{\prime}\right)^{2}+\left(\gamma_{i}+z+z^{\prime}\right)^{2}}, K_{0}(\cdot)$ is the zeroth-order modified Bessel function of the second kind, and $H_{0}^{(2)}(\cdot)$ is the zeroth-order Hankel function of the second kind. Taking into account that both the quasi-static term $\tilde{S}_{0}$ and the first term in (4) can be considered as particular cases of the exponential terms in expansion (9) (when $\gamma_{i}=0$ ), its spatial-domain counterpart can be readily obtained from (15).

Looking at (15), the first option, i.e., $\operatorname{Im}\left(k_{x 0}\right)<0$, gives place to exponentially decaying fields in the normal $z$-direction, a situation that is compatible with the bound regime. The second option in (15) provides exponentially growing fields in the upper half-space, thus accounting for the space-wave leaky regime. The two-valued (15) can be related to the different integration paths used in the SDA of space-wave leaky modes [3], [16]. The conventional real-axis $C_{0}$ integration path in Fig. 3 is found to be equivalent to the $\operatorname{Im}\left(k_{x 0}\right)<0$ choice in (15), whereas the $C_{2}$ integration path in this figure would reflect the $\operatorname{Im}\left(k_{x 0}\right)>0$ choice.

\section{Solving the Integral Equation}

A relevant feature of the present approach is that very accurate closed-form expressions are obtained for the kernels of the integral equation for both the bound and leaky regimes. The regime to be studied (bound, surface wave, and space wave) is simply selected by the convenient choice in (12) and (15). This provides a systematic, very efficient, and rather simple way to 
study the propagation phenomena, superseding the possible inconveniences of the SDA related to the numerical evaluation of the spectral integrals along different integration paths in the complex plane.

Once the space-domain kernels are obtained, the integral equation is solved by using the Galerkin moment method. Chebyshev polynomials of the first and second kinds weighed by the edge condition are used as basis functions to analyze structures with infinitely thin conductors. Structures with arbitrary cross-sectional conductors are analyzed employing nonuniformly distributed piecewise linear functions as basis functions. In both cases, a quasi-analytical evaluation of the matrix entries is carried out. More details can be found in [12] and [14].

\section{NUMERICAL RESULTS}

The present method has been validated by comparing our results with previously published results and with those obtained by means of a well-established SDA code [19] developed for zero-thickness strip-like structures. The agreement for both bound and leaky regimes is excellent for all the cases considered. As a first example, Fig. 4(a) and (b) shows the normalized phase and attenuation constants for a space-wave leaky mode supported by a microstrip line previously studied by Michalsky and Zheng [7] only for the zero-thickness case. The comparison between the data reported in [7] with our MPIE-DCIT results shows an excellent agreement. Fig. 4(a) and (b) also includes results for two nonzero thickness cases, i.e., rectangular and trapezoidal cross-sectional conductors. These curves show how the shape of the conductor can influence the dispersion curves. To give an idea of the overall computational effort required by our method, 100 values of the propagation constant for the zero-thickness case were computed in $6 \mathrm{~s}$ with a Pentium II $450-\mathrm{MHz}$ computer. Rectangular or trapezoidal structures require a few seconds to get a single value of $k_{y}$ using the same platform. Fig. 5 shows another comparison with the dispersion curves of a circular-wire transmission line reported in [7, Fig. 7]. Again, the agreement found both for the bound (EH0) and the space-wave leaky (EH1) modes is very good. It is interesting to mention that, in the computation of the above results, because of the presence of several slab waveguide modes (TM0, TE1, and TM1), the explicit contribution of up to three surface-wave poles had to be considered in the calculus.

Finally, and to show the capability of our method to deal with multiconductor and multilayer nonplanar structures, a two-layer LIGA micromachined transmission line has been analyzed. The LIGA process, described in [20], gives rise to metallizations with a high thickness/width ratio. This makes it possible to use the conductor thickness as a new variable in the design of filters and couplers. The analyzed structure consists of a pair of square cross-sectional conductors $(200 \times 200 \mu \mathrm{m})$ separated by a $120-\mu \mathrm{m}$ distance and placed on a layered substrate. The dispersion curves of the normalized propagation and attenuation constants for a surface-wave leaky mode appearing in this structure are shown in Fig. 6. To study the effect of the metallization thickness, the dispersion curves for the same structure assuming zero-thickness conductors are also shown. It can be seen

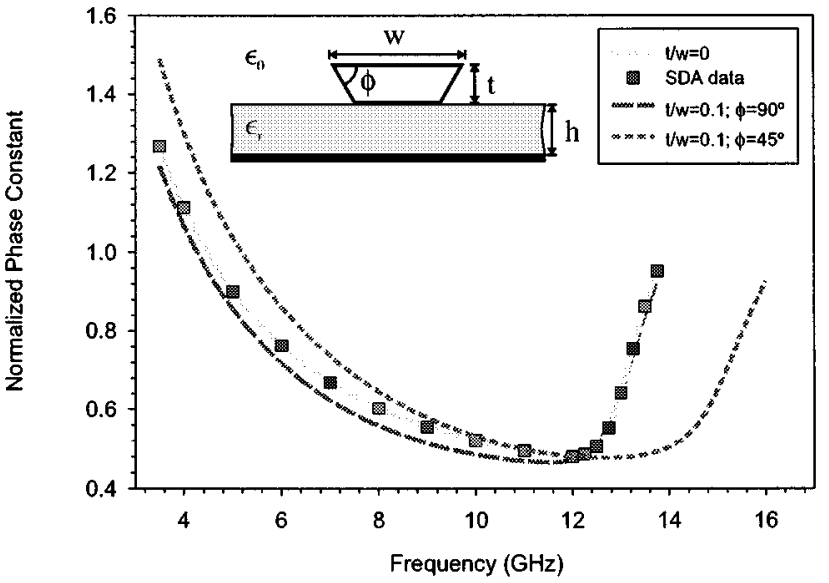

(a)

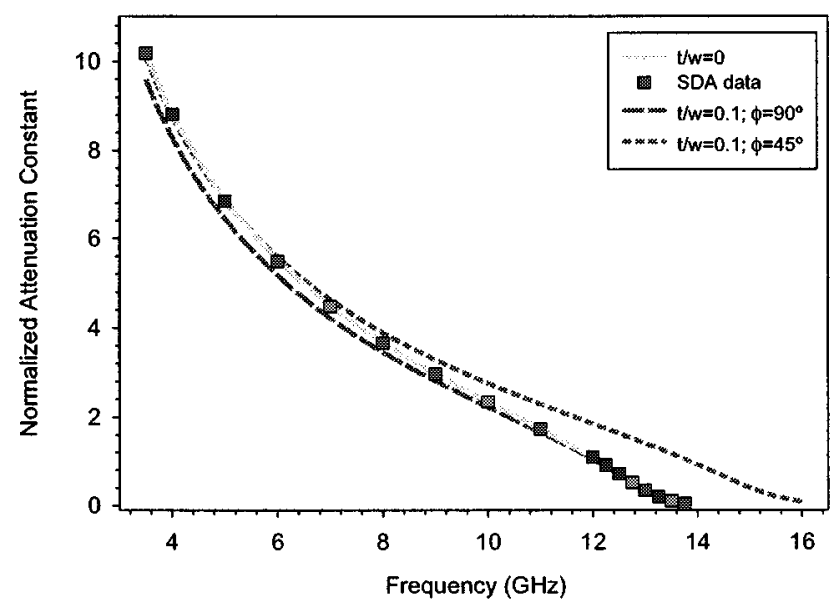

(b)

Fig. 4. Normalized: (a) phase and (b) attenuation constant for a space-wave leaky mode in the microstrip line shown in the inset: $\varepsilon_{r}=9.8, w=3 \mathrm{~mm}$, $h=0.635 \mathrm{~mm}$.

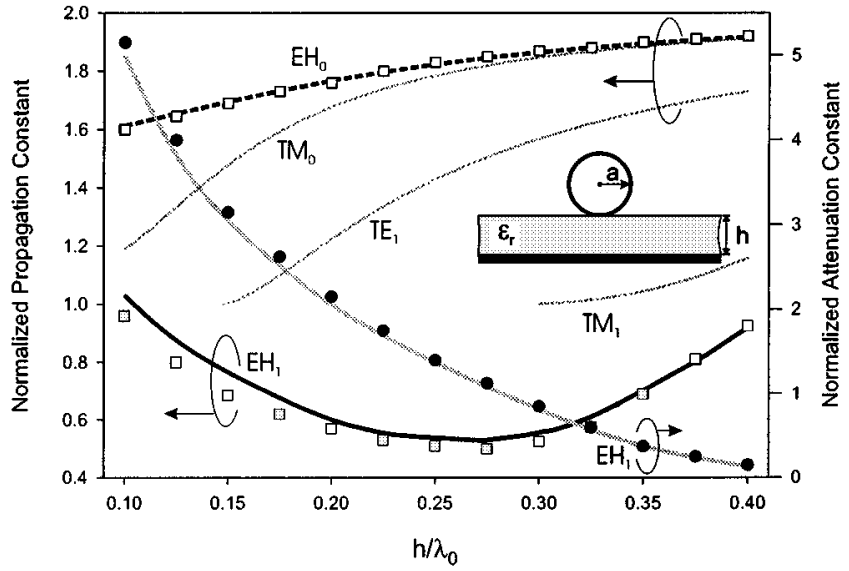

Fig. 5. Normalized phase and attenuation constants of the fundamental mode and the first higher mode in a circular-wire transmission line. Lines: our results. Symbols: data in [7]. Structural parameters: $a / h=0.25, \varepsilon_{r}=4$.

that the splitting point (the point where a complex leaky solution merges with a complex conjugate solution, and the two solutions then split apart as two improper real modes) appears at lower frequencies in the nonplanar case. Results for the square-shape conductors have been obtained with 15 basis function over each 


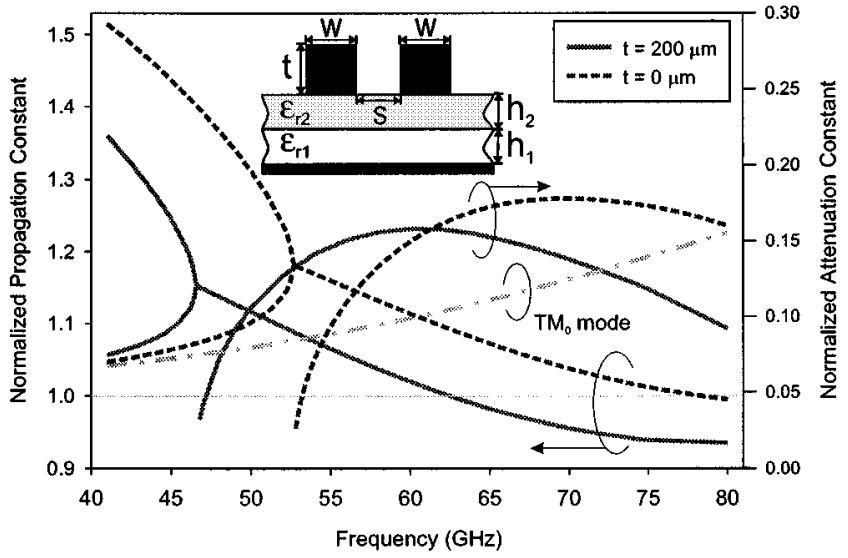

Fig. 6. Normalized phase and attenuation constants for a surface-wave leaky mode in a two-conductor and two-layer transmission line with $w=200 \mu \mathrm{m}$, $s=120 \mu \mathrm{m}, h_{1}=h_{2}=210 \mu \mathrm{m}, \varepsilon_{r 1}=3$, and $\varepsilon_{r 2}=4.6$.

conductor. The CPU time was much larger for the nonplanar case because of the use of many basis functions (in comparison with the zero-thickness strip case) and the need for computing more time-consuming integrals. Regardless, the method provides the possibility of efficiently determining in a quantitative way the influence of the thickness or the shape of the conductors in the leaky regime.

\section{CONCLUSIONS}

A new approach based on the combination of the MPIE and DCIT has been developed to compute in a simple and systematic way the propagation characteristics of both bound, surface-, and space-wave leaky modes in printed-circuit lines. One of the main goals of this approach has been the obtaining of accurate expressions for the kernels of the corresponding integral equation in a quasi-analytical way. This has avoided the involved task of integrating along diverse integration paths in the transverse wavenumber complex plane required in the SDA. The use of the complex images technique has also provided high accuracy and efficiency to the analysis. Working in the spatial domain has allowed for a direct (although nontrivial) extension of the analysis to nonplanar conductors. Some results have been reported to show the accuracy and efficiency of the computer code developed.

\section{APPENDIX}

An efficient method to compute integral (11) and its derivative with respect to $\left|x-x^{\prime}\right|$ will be shown here. To simplify the notation, the following new variables will be used: $\chi=\left|x-x^{\prime}\right|$ and $\zeta=\left(z+z^{\prime}\right)$.

The integrand in (11) has two poles at $k_{x}= \pm k_{x p}$ and two branch points at $k_{x}= \pm k_{x 0}$. If an integration path running from $-\infty$ to $\infty$ along the real axis is conveniently closed in the lower half-plane, the result of this integral is determined by the surface-wave pole enclosed by such a path. Considering that the closed path must detour around the branch cut, (11) can be expressed as

$$
I_{p}=\frac{e^{-j k_{x p} \chi}}{j k_{x p}} e^{-j k_{z p} \zeta}+\Upsilon_{p}
$$

where

$$
\begin{aligned}
& \Upsilon_{p}=-2 \operatorname{sign}[\left.\operatorname{Re}\left(k_{x 0}\right)\right] e^{-j k_{x 0} \chi} \\
& \times \int_{0}^{\infty} \frac{\sin \left(\sqrt{s^{2}+2 j k_{x 0} s} \zeta\right)}{s^{2}+2 j k_{x 0} s-k_{z p}^{2}} e^{-s \chi} d s .
\end{aligned}
$$

The first term in (16) is a two-valued function accounting for the surface-wave pole contribution with $\operatorname{Im}\left(k_{z p}\right)<0$-as in (12), the sign of $\operatorname{Im}\left(k_{x p}\right)$ determines the bound/leaky regime under consideration. The second term in (16) gives the contribution of the integration along the branch cut (assuming that $\kappa_{p}>k_{0}$ ) and, more specifically, it comes from an integration along a vertical branch cut with $\operatorname{Im} \sqrt{s^{2}+2 j k_{x 0} s}<0$. As happens in (15), the sign of $\operatorname{Im}\left(k_{x 0}\right)$ in (17) sets the regime (bound/space-wave leaky) to be treated. The change of variable $k_{x}=k_{x 0}-j s$ causes integral $\Upsilon_{p}$ to be quickly calculated by using, for instance, Gauss quadratures.

The derivative of $I_{p}$ can be readily computed from the above expressions to give

$$
\frac{\partial I_{p}}{\partial \chi}=-\pi e^{-j k_{x p} \chi} e^{-j k_{z p} \zeta}+\frac{\partial \Upsilon_{p}}{\partial \chi}
$$

where

$$
\begin{aligned}
\frac{\partial \Upsilon_{p}}{\partial \chi}=-j k_{x 0} \Upsilon_{p}+2 \operatorname{sign}\left[\operatorname{Re}\left(k_{x 0}\right)\right] e^{-j k_{x 0} \chi} & \\
& \times \int_{0}^{\infty} \frac{\sin \left(\sqrt{s^{2}+2 j k_{x 0} s} \zeta\right)}{s^{2}+2 j k_{x 0} s-k_{z p}^{2}} s e^{-s \chi} d s .
\end{aligned}
$$

Note that, for large values of $\zeta$, the integrands in (17) and (19) become highly oscillatory, thus giving rise to numerical drawbacks. In that case, $I_{p}$ can be calculated by means of a direct integration of (11). This numeric integration must be performed along path $C_{0}$ in Fig. 2 to account for the bound regime, whereas path $C_{1} / C_{2}$ must be chosen if we are looking for a surface-/space-wave leaky regime.

The integral along the $C_{0}$ real-axis path, denoted as $I_{p}^{C_{0}}$, can be rewritten in the following form:

$$
I_{p}^{C_{0}}=2 \int_{0}^{\infty} \frac{\cos \left(k_{x} \chi\right)}{k_{x}^{2}-k_{x p}^{2}} e^{-j k_{z 0} \zeta} d k_{x}
$$

$\left(\operatorname{Im}\left(k_{z 0}\right)<0\right)$, which can be efficiently calculated by using Gauss quadratures. Note that large values of $\zeta$ lead to a fast convergence for $I_{p}^{C_{0}}$.

The integration along path $C_{1}$, i.e., $I_{p}^{C_{1}}$, can be split into integration along the real axis $\left(C_{0}\right)$ in addition to integration along $C_{p 1}$ and $C_{p 2}$ paths in Fig. 2, namely,

$$
I_{p}^{C_{1}}=I_{p}^{C_{0}}+I_{p}^{C_{p 1}}+I_{p}^{C_{p 2}}
$$

where

$$
I_{p}^{C_{p 1}}+I_{p}^{C_{p 2}}=\frac{2 \pi}{j k_{x p}} \cos \left(j k_{x p} \chi\right) e^{-j k_{z p} \gamma}
$$

with $\operatorname{Im}\left(k_{z p}\right)<0$ and $\operatorname{Im}\left(k_{x p}\right)>0$.

According to [16], the integral along path $C_{2}$, i.e., $I_{p}^{C_{2}}$, can be expressed as integral (21) plus an integral running along a loop, as shown in [16]

$$
I_{p}^{C_{2}}=I_{p}^{C_{1}}+I_{p}^{\mathrm{loop}}
$$


where

$$
I_{p}^{\mathrm{loop}}=\frac{2 j}{k_{x 0}} \int_{-1}^{1} \frac{\sin \left(k_{x 0} \sqrt{1-u^{2} \zeta}\right)}{u^{2}-\left(\frac{k_{x p}}{k_{x 0}}\right)^{2}} e^{-j k_{x 0} u \chi} d u
$$

with $\operatorname{Im}\left(k_{x 0}\right)>0$ and $\operatorname{Im}\left(k_{x 0} \sqrt{1-u^{2}}\right)<0$.

The derivative of $I_{p}$ with respect to $\chi=\left|x-x^{\prime}\right|$ can be readily obtained from (20) and (22) yielding the following expressions:

$$
\begin{aligned}
\frac{\partial I_{p}^{C_{0}}}{\partial \chi} & =-2 \int_{0}^{\infty} k_{x} \frac{\sin \left(k_{x} \chi\right)}{k_{x}^{2}-k_{x p}^{2}} e^{-j k_{z 0} \zeta} d k_{x} \\
\frac{\partial I_{p}^{C_{1}}}{\partial \chi} & =\frac{\partial I_{p}^{C_{0}}}{\partial \chi}+2 \pi \sin \left(k_{x p} \chi\right) e^{-j k_{z p} \zeta} \\
\frac{\partial I_{p}^{\text {loop }}}{\partial \chi} & =2 \int_{-1}^{1} u \frac{\sin \left(k_{x 0} \sqrt{1-u^{2} \zeta}\right)}{u^{2}-\left(\frac{k_{x p}}{k_{x 0}}\right)^{2}} e^{-j k_{x 0} u \chi} d u .
\end{aligned}
$$

\section{REFERENCES}

[1] D. Mirshekar-Syahkal, Spectral Domain Method for Microwave Integrated Circuits. Norwood, MA: Artech House, 1990.

[2] J. Boukamp and R. H. Jansen, "Spectral domain investigation of surface wave excitation and radiation by microstrip lines and microstrip disk resonators," in Proc. 13th Eur. Microwave Conf., Sept. 1983, pp. 721-726.

[3] N. K. Das and D. M. Pozar, "Full-wave spectral-domain computation of material, radiation, and guided wave losses in infinite multilayered printed transmission lines," IEEE Trans. Microwave Theory Tech., vol. 39, pp. 54-63, Jan. 1991.

[4] F. Mesa, R. Marqués, and M. Horno, "An efficient numerical spectral domain method to analyze a large class of nonreciprocal planar transmission lines," IEEE Trans. Microwave Theory Tech., vol. 40, pp. 1630-1641, Aug. 1992.

[5] D. Nghiem, J. T. Williams, D. R. Jackson, and A. A. Oliner, "Proper and improper dominant mode solutions for a stripline with an air gap," Radio Sci., vol. 28, no. 6, pp. 1163-1180, Nov.-Dec. 1993.

[6] C.-I. G. Hsu, R. F. Harrington, K. A. Michalski, and D. Zheng, “Analysis of multiconductor transmission lines of arbitrary cross section in multilayered uniaxial media," IEEE Trans. Microwave Theory Tech., vol. 41, pp. 70-78, Jan. 1993.

[7] K. A. Michalsky and D. Zheng, "Rigorous analysis of open microstrip lines of arbitrary cross section in bound and leaky regimes," IEEE Trans. Microwave Theory Tech., vol. 37, pp. 2005-2010, Dec. 1989.

[8] D. G. Fang, J. J. Yang, and G. Y. Delisle, "Discrete image theory for horizontal electric dipoles in a multilayered medium," Proc. Inst. Elect. Eng., vol. 135, pp. 297-302, Oct. 1988.

[9] Y. L. Chow, J. J. Yang, D. G. Fang, and G. E. Howard, "A closed-form spatial Green's function for the thick microstrip substrate," IEEE Trans. Microwave Theory Tech., vol. 39, pp. 588-592, Mar. 1991.

[10] M. I. Aksun, "A robust approach for the derivation of closed-form Green's functions," IEEE Trans. Microwave Theory Tech., vol. 44, pp. 651-658, May 1996.

[11] R.-C. Hsieh and J.-T. Kuo, "Evaluation of spatial Green's functions for microstrips: Fast Hankel transform algorithm and complex image method," Electron. Lett., vol. 34, no. 11, pp. 1110-1111, May 1998

[12] J. Bernal, F. Medina, R. R. Boix, and M. Horno, "Fast full wave analysis of multistrip transmission lines based on MPIE and complex images," IEEE Trans. Microwave Theory Tech., vol. 48, pp. 445-452, Mar. 2000.

[13] E. A. Soliman, P. Pieters, E. Beyne, and G. A. E. Vandenbosch, "Numerically efficient spatial-domain moment method for multislot transmission lines in layered media-Application to multislot in MCM-D technology," IEEE Trans. Microwave Theory Tech., vol. 47, pp. 1782-1787, Sept. 1999.

[14] J. Bernal, F. Medina, R. R. Boix, and M. Horno, "Full-wave analysis of nonplanar transmission lines on layered medium by means of MPIE and complex image theory," IEEE Trans. Microwave Theory Tech., vol. 49, pp. 177-185, Jan. 2001.
[15] C. Di-Nallo, F. Mesa, and D. R. Jackson, "Excitation of leaky modes on multilayer stripline structures," IEEE Trans. Microwave Theory Tech., vol. 46, pp. 1062-1071, Aug. 1998.

[16] F. Mesa, C. Di-Nallo, and D. R. Jackson, "The theory of surface-wave and space-wave leaky-mode excitation on microstrip lines," IEEE Trans. Microwave Theory Tech., vol. 47, pp. 207-215, Feb. 1999.

[17] A. A. Oliner, "Leakage from higher modes on microstrip line with application to antennas," Radio Sci., vol. 22, no. 6, pp. 907-912, Nov. 1987.

[18] D. G. Dudley, Mathematical Foundations for Electromagnetic Theory. Piscataway, NJ: IEEE Press, 1994.

[19] R. Marqués and F. Mesa, "Spectral domain analysis of higher order leaky modes in microstrip lines: A new spectral-gap effect," J. Electromagn. Waves Applicat., vol. 11, pp. 1367-1384, 1997.

[20] T. L. Willke and S. S. Gearhart, "LIGA micromachined planar transmission lines and filters," IEEE Trans. Microwave Theory Tech., vol. 45, pp. 1681-1688, Oct. 1997.

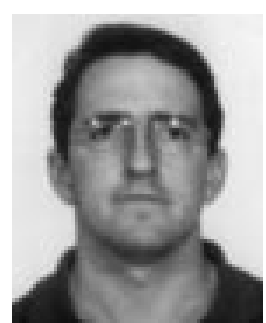

Joaquín Bernal was born in Seville, Spain, in 1971. He received the Licenciado and Doctor degrees from the University of Seville, Seville, Spain, in 1994 and 2000 , both in physics.

In 1995, he joined the Department of Electronic and Electromagnetism, University of Seville. Since 1998, he has been an Assistant Professor in the Department of Applied Physics III at the same university. His research interests focus on the analysis of planar structures for integrated microwave circuits and high-speed very large scale integration (VLSI)

interconnects.

Dr. Bernal was the recipient of a Junta de Andalucía scholarship.

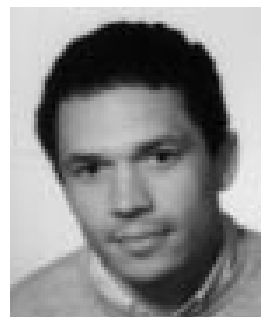

Francisco Mesa (M’94) was born in Cádiz, Spain, on April 1965. He received the Licenciado and Doctor degrees from the University of Seville, Seville, Spain, in 1989 and 1991, respectively, both in physics.

$\mathrm{He}$ is currently an Associate Professor in the Department of Applied Physics I, University of Seville. His research interest focuses on electromagnetic propagation/radiation in planar lines with general anisotropic materials.

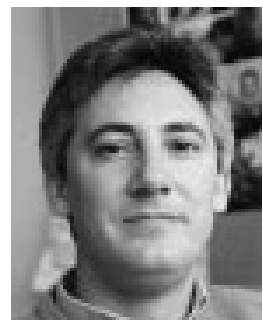

Francisco Medina (M'90-SM'01) was born in Puerto Real, Cádiz, Spain, in November 1960. He received the Licenciado and Doctor degrees from the University of Seville, Seville, Spain, in 1983 and 1987 , respectively, both in physics.

From 1986 to 1987, he spent the academic year with the Laboratoire de Microondes de l'ENSEEIHT, Toulouse, France. From 1985 to 1989, he was a Profesor Ayudante (Assistant Professor) with the Department of Electronics and Electromagnetism, University of Seville, and since 1990, he has been a Profesor Titular (Associate Professor) of electromagnetism. He is also currently Head of the Microwaves Group, University of Seville. His research interest includes analytical and numerical methods for guidance, resonant and radiating structures, passive planar circuits, and the influence on these circuits of anisotropic materials.

Dr. Medina was a member of both the Technical Program Committee (TPC) of the 23rd European Microwave Conference, Madrid, Spain, 1993, and the TPC of ISRAMT'99, Malaga, Spain. He is on the Editorial Board of the IEEE TRANSACTIONS ON MICROWAVE THEORY AND TECHNIQUES. He has been a reviewer for other IEEE and Institution of Electrical Engineers (IEE), U.K., publications. 